\title{
Methode zur gezielten Identifikation von Marktunsicherheiten zur Unterstützung der Zielsystementstehung
}

\section{A methodology for the systematic identification of market uncertainties to support the development of the system of objectives}

\author{
Valentin Zimmermann ${ }^{1 *}$, Rudi Prinz ${ }^{1}$, Albert Albers ${ }^{1}$ \\ ${ }^{1}$ Institute of Product Engineering, Karlsruhe Institute of Technology \\ ${ }^{*}$ Korrespondierender Autor: \\ Valentin Zimmermann \\ Kaiserstraße 10 \\ 76131 Karlsruhe \\ http://www.ipek.kit.edu/ \\ Telefon: +49721 608-42371 \\ Mail: valentin.zimmermann@partner.kit.edu
}

\begin{abstract}
Product development and the associated processes are characterized by uncertainties. Thus, the elements of the system of objectives are also subject to uncertainties, which influence the product, its development and its success on the market. The systematic handling of these uncertainties is crucial for the success of product development. Uncertainty can be reduced by validation. However, validation has only a low priority, since, among other things the awareness of the necessity of validation and the uncertainties is only partly existing. In the course of this publication, a method to systematically evaluate the elements of the system of objectives concerning market uncertainty to increase the awareness of the uncertainties and to support the planning of validation activities is proposed.
\end{abstract}

\section{Keywords}

System of Objectives, Product Engineering, Validation, Market Uncertainty 


\section{Motivation}

Viele Produktentwicklungsprojekte halten den angedachten Zeitrahmen nicht ein, überschreiten das geplante Budget, erfüllen die gesetzten Ziele nur unzureichend oder die Produkte erzielen schließlich nur bedingt einen Erfolg am Markt [1]. Grund hierfür sind insbesondere Unsicherheiten, die in der Entwicklung auftreten und beispielsweise die Stabilität von Zielen und Anforderungen beeinflussen [2]. Der Anstieg dieser Unsicherheiten in der Produktentwicklung ist insbesondere auf eine Verkürzung der Entwicklungsdauer, da die Märkt nach immer mehr Produkten in kürzerer Zeit verlangen, auf eine ansteigende Marktdynamik durch neue Wettbewerber und auf einen Anstieg der Komplexität, zurückzuführen [u.a. 3, 4]. Die Komplexität der Produkte und zugehörigen Entwicklungsprozesse steigt insbesondere deswegen an, da in der Mechatroniksystementwicklung eine Verschiebung der Marktleistungen von autarken physischen Produkten hin zu hybriden Leistungsbündel mit Dienstleistungs- und Softwareanteil stattfindet [5]. Um mit diesen ansteigenden Unsicherheiten umzugehen bedarf es einen frühen und kontinuierlichen Erkenntnisgewinn, wie es beispielsweise die agilen Ansätze mit Ursprung in der Softwarebranche durch ihr iteratives Vorgehen fördern [6]. Sowohl die frühe Fokussierung hinsichtlich der Kunden- und Anwenderbedürfnisse als auch die kontinuierliche Validierung steigern das Potential der Entwicklungsprojekte hinsichtlich ihres erfolgreichen Abschlusses [4]. Eine Implementierung dieser agilen Ansätze ist auch für die Mechatroniksystementwicklung von Relevanz, allerdings lassen sich diese, unter anderem aufgrund der Rahmenbedingungen der physischen Produktentwicklung, nicht einfach übernehmen [7]. So hat die Validierung in der Mechatroniksystementwicklung beispielsweise einen geringen Stellenwert, bzw. das Bewusstsein für die Notwendigkeit dieser und die dahinterstehenden Unsicherheiten ist nicht derart ausgeprägt [8]. Deshalb gilt es gezielt das Bewusstsein der Produktentwickler für die Unsicherheiten und den Umgang damit zu schärfen, und systematisch die Integration von Validierungsaktivitäten in die Entwicklungsprozesse zu unterstützen.

\section{Stand der Forschung}

\subsection{Produktentwicklung als iterativer Prozess}

Die Produktentwicklung lässt sich nach ALBERS [9] entsprechend des ZHO-Systemtripels durch die Überführung eines zunächst vagen initialen Zielsystems in ein Objektsystem durch das Handlungssystem beschreiben. Dabei beschreibt das Handlungssystem ein soziotechnisches System, welches sämtliche Methoden, Aktivitäten und Prozesse, sowie die zur Umsetzung nötigen Ressourcen umfasst. Das Zielsystem umfasst die an das Produkt gestellten Ziele, Anforderungen und Randbedingungen sowie deren Wechselwirkung. Das Zielsystem wird im Verlauf der Produktentwicklung ständig erweitert und konkretisiert. So können sich im Verlaufe der Produktentwicklung Ziele verändern, neue Ziele entstehen oder Ziele obsolet werden. Das Objektsystem beinhaltet sämtliche in der Entwicklung entstehenden Objekte, Lösungen und das endgültige Produkt. Ziel- und Objektsystem stehen jedoch nicht direkt in Kontakt, sondern vielmehr verbindet das Handlungssystem die beiden miteinander und überführt durch Analyse und Synthese das Ziel- in das Objektsystem. Dieser Prozess ist jedoch keinesfalls ein sequenziell ablaufender Prozess, sondern vielmehr ein iterativer Prozess, indem durch die Analyse bestehender Ziele und Objekte der Lösungsraum eingeschränkt bzw. die Wissensbasis erweitert wird und darauffolgend Synthesetätigkeiten zur Generierung neuer Ziele bzw. Objekte abgeleitet werden. [10] Um aus dem Zielsystem Entwicklungsaktivitäten ableiten zu können führen ALBERS ET AL. Dimensionen zur Bewertung der Zielsystemelemente ein [10]. Diese sind Härtegrad, Auswirkung, Beeinflussbarkeit und Reifegrad. Der Härtegrad beschreibt die Änderbarkeit eines Zielsystemelements und die Auswirkung eines Zielsystemelements beschreibt dieses hinsichtlich Zeit- und, Kostenaufwand der Umsetzung und der Än- 
derung. Die Beeinflussbarkeit umfasst die Fähigkeit einzelner Akteure und Organisationseinheiten ein Zielsystemelement zu ändern oder zu definieren. Der Reifegrad eines Zielsystemelements spiegelt die vorliegende Unsicherheit des Zielsystems wieder. [10]

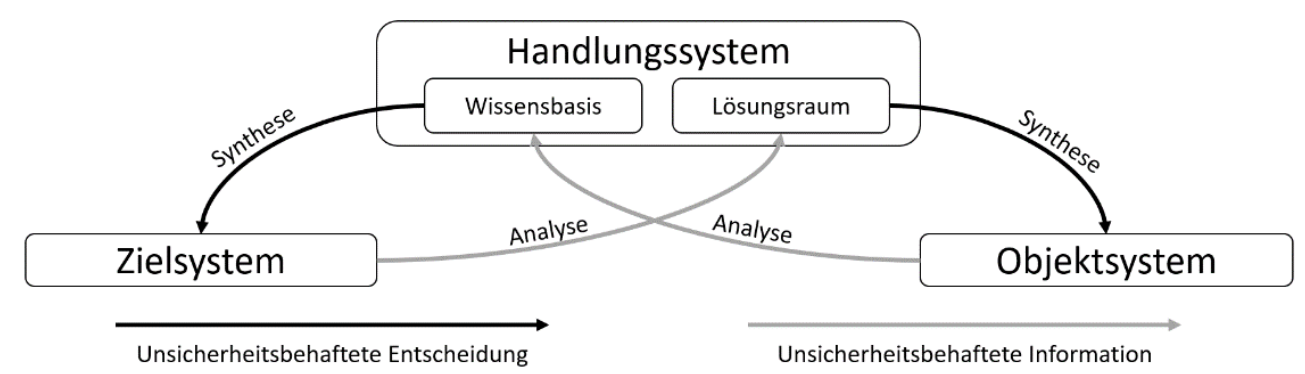

Abbildung 1: Erweitertes ZHO-Systemtripel unter Einfluss von Unsicherheit [10] S. 257

\subsection{Unsicherheit in der Produktentwicklung}

DE WECK ET AL. beschreiben Unsicherheit sowohl als die Wahrscheinlichkeit, dass während der Produktentwicklung getroffenen Annahmen falsch sind, als auch das Auftreten völlig unbekannter Fakten, die Einfluss auf die Entwicklung des Produkts, das Produkt selbst oder den Erfolg des Produkts am Markt haben. Dabei kann die Einflussnahme der entsprechenden Unsicherheit sowohl negativ als auch positiv sein. [11] Nach EBERT sind Anforderungen per Definition mit Unsicherheiten behaftet, die den Erfolg eines Produktes potentiell beeinflussen [12]. ALBERS [9] beschreibt, dass die Unsicherheit von Zielsystemelementen gegenläufig zu deren Reifegrad verläuft. Zur Operationalisierung der Bewertung des Reifegrades bzw. der Unsicherheit bauen ALBERS ET AL. [10] auf das Unsicherheitsverständnis nach HASTINGS und MCMANUS [13] auf und beschreiben sie als Wissens- und Definitionslücken. Dabei beschreiben Wissenslücken fehlendes oder unzureichendes Wissen, während Definitionslücken ausstehende Entscheidungen oder Spezifizierungen umfassen. Tiefergehend unterteilen sie Unsicherheit in statistisch charakterisierbare Unsicherheiten, die aufgrund empirisch verteilter Zustände vorherrschen, in bekannte Unsicherheiten, deren Existenz bekannt ist, und unbekannte Unbekannte bzw. Unsicherheiten, wobei hier kein Wissen bezüglich der Existenz dieser vorliegt. WYNN [14] unterscheidet dahingehend in epistemische Unsicherheiten, Wissens- und Definitionslücken, die beispielsweise durch Test geschlossen werden können, und aleatorische Unsicherheiten bei denen dies nicht möglich ist. Im Kontext der Produktentwicklung wird bei Unsicherheiten häufig zwischen Technologie- und Marktunsicherheiten unterschieden [15]. Technologieunsicherheit beschreibt Wissens- und Definitionslücken hinsichtlich der technischen Machbarkeit der Produkte [16], wohingegen sich Marktunsicherheit auf entsprechende Lücken hinsichtlich der Zielmärkte [4], Kundenbedürfnisse [16], Zahlungsbereitschaft [17] und des Anwendungsfalls [18] beläuft. BROWNING [2] beschreibt, dass insbesondere Marktunsicherheiten die Qualität von Anforderungen und Zielen beeinflussen. Je besser Kunde, Anwender und die Randbedingungen verstanden werden, umso stabilere Ziele und Anforderungen können formuliert werden und so beispielsweise spätere Änderungen vermieden werden [2].

\subsection{Ansätze zum Umgang mit Unsicherheit}

In der Produktentwicklung können vorhandene Unsicherheiten einen großen Einfluss auf das Produkt, die Entwicklung oder den Erfolg des Produktes am Markt haben. Deshalb ist ein systematischer Umgang mit den Unsicherheiten anzustreben, um damit die verbundenen Risiken zu minimieren und die Chancen zu erhöhen. [4] Die Validierung ist die zentrale Aktivität zur Generierung von Wissen und damit zur Reduzierung von Unsicherheit [9]. Jedoch gilt es zunächst zu identifizieren, welche Zielsystemelemente mit Unsicherheiten behaftet sind. Ein möglicher Ansatz hierbei stellt die Fehlermöglichkeits- und Einflussanalyse (FMEA) [u.a. 19] 
dar. Ziel dieser ist es sämtliche potentielle Ausfälle eines Systems zu ermitteln. Dazu werden auf Basis einer Struktur und Funktionsanalyse sämtliche potentielle Fehler ermittelt und entsprechende Fehlerursachen und Fehlerfolgen zugeordnet. Abschließend werden die Auftretenswahrscheinlichkeit, die Bedeutung und die Erkennungswahrscheinlichkeit des Fehlers bewertet, darauf aufbauend die Risikoprioritätszahl ermittelt und Maßnahmen zur Fehlervermeidung bestimmt [u.a. 19]. In Anlehnung an die FMEA fokussiert die UMEA - Uncertainty-Mode and Effect-Analysis [20] eine Analyse der vorliegenden Unsicherheiten. Zielsetzung ist es Unsicherheit im Produktlebenszyklus zu analysieren und abzuschätzen. In der UMEA wird Unsicherheit als Einfluss auf gegebene Variablen verstanden und die Auswirkung dieses analysiert. ALBERS ET AL. [18] bewerten zur Priorisierung von Validierungsaktivitäten die Zielsystemelemente entsprechend der Zielsystemdimensionen und bestimmen so die entsprechende Kritikalität. Den Fokus setzen sie hierbei auf die Bekanntheit der Anwendung und der Technologie, um Schlüsse hinsichtlich des Reifegrades zur erhalten [18] und betrachten somit explizit Technologieunsicherheiten.

\section{Forschungsdesign}

Produktentwickler werden bei der Entwicklung mechatronischer Systeme mit Unsicherheiten konfrontiert. So sind per Definition sämtliche Ziele und Anforderungen mit Unsicherheiten behaftet [12], die das Produkt, dessen Entwicklung und den Markterfolg beeinflussen können [11]. Um sie im Umgang mit den Unsicherheiten zu unterstützen, soll der frühe und kontinuierliche Erkenntnisgewinn gefördert werden. Die Validierung ist die zentrale Aktivität, um Wissen zu generieren bzw. Wissenslücken zu schließen und damit Unsicherheiten zu reduzieren [9]. Der Stellenwert der Validierung in der industriellen Praxis ist jedoch nicht derart ausgeprägt, wie beispielsweise in den agilen Ansätzen. Gründe dafür sind neben mangelnden Ressourcen häufig auch das mangelnde Bewusstsein der Produktentwickler für Marktunsicherheiten und die Notwendigkeit der Validierung. Weiter ist die Schwierigkeit der Identifikation unsicherheitstreibender und damit zu validierenden Zielsystemelemente ein Hemmnis. Somit bedarf es einer Methode, die Produktentwickler im Umgang mit Marktunsicherheiten unterstützt. Dazu gilt es das Bewusstsein der Produktentwickler für die Marktunsicherheiten mit denen Zielsystemelemente behaftet sind, zu erhöhen, und die Produktentwickler dabei zu unterstützen die kritischen Zielsystemelemente zu identifizieren, um Validierungsaktivitäten abzuleiten und zu planen. Da Unsicherheit als solche nicht objektiv bewertbar ist [21], gilt es eine Metrik zu erarbeiten, die Produktentwickler die Unsicherheit zugänglich macht. Dazu werden im Rahmen dieser Veröffentlichung die nachfolgenden Forschungsfragen beantwortet.

- Wie lässt sich Marktunsicherheit, mit welcher Zielsystemelemente behaftet sind, auf Basis von Einflussfaktoren charakterisieren?

- Wie ist eine Methode zur gezielten Identifikation von mit Marktunsicherheiten behafteten Zielsystemelementen zu gestalten?

- Welchen Beitrag leistet die Methode bei der systematischen Identifikation unsicherheitsbehafteter Zielsystemelemente?

Zunächst wurde eine Literaturrecherche durchgeführt, im Zuge derer 395 Einflussfaktoren hinsichtlich des Themenfeldes Marktunsicherheit identifiziert wurden. Um die Einflussfaktoren zu strukturieren, wurden diese in einem ersten Schritt gefiltert und daraufhin mithilfe einer Design Structure Matrix (DSM) die Wirkzusammenhänge der Faktoren ermittelt. Dabei wurden die Faktoren hinsichtlich ihrer gegenseitigen Beeinflussung gegeneinander gewichtet. So konnten die Hauptdimensionen abgeleitet werden. Stark verwandte Faktoren wurden zu Faktorclustern zusammengefasst. Um eine einheitliche Bewertung der Faktoren zu ermöglichen, wurden jedem Faktor Ausprägungsformen zugeteilt. Weiter wurde ein Rechenmodell erstellt, 
das den Einfluss der einzelnen Faktoren und Faktorcluster auf die Dimensionen abbildet. Abschließend wurde die erarbeitete Methode in einem Softwaretool umgesetzt, um sie den Methodenanwendern zugänglich zu machen. Unter Verwendung dieses Tools wurde die Methode im Rahmen eines Entwicklungsprojektes in der Hekatron Vertriebs $\mathrm{GmbH}$ evaluiert. Dabei wurde eine Anwendungs- und Erfolgsevaluierung entsprechend der Design Research Methodology nach BLESSING und CHAKRABARTI [22] durchgeführt. Mithilfe einer Befragung vor und nach der Anwendung konnte das Bewusstsein und die Wahrnehmung der Unsicherheit der Methodenanwender überprüft werden. Zusätzlich wurden Erfolgsfaktoren definiert, wodurch die Eignung der Methode zur Unterstützung beim Umgang mit Marktunsicherheit festgestellt werden konnte.

\section{Ergebnisse}

\subsection{Charakterisierung von Marktunsicherheit in der Produktentwicklung}

Zunächst wurde eine Literaturrecherche zum Themenfeld Marktunsicherheit durchgeführt und dabei Einflussfaktoren, mit direktem Zusammenhang zur Produktentwicklung, welche Marktunsicherheit charakterisieren, identifiziert. Dabei wurde eine Sammlung mit 395 Faktoren aufgestellt. Diese wurden nachfolgend gefiltert. Faktoren, welche sich nur subjektiv bewerten lassen, genau wie Faktoren deren direkter Zusammenhang zur Unsicherheit nur bedingt bekannt ist oder bei denen der Zusammenhang zwischen der Ausprägung des Faktors und der Auswirkung - verstärkend oder abschwächend - auf die Marktunsicherheit nicht bekannt ist, wurden aus der Liste entfernt. Mithilfe einer Design Structure Matrix (DSM) wurden die Wirkzusammenhänge der verbliebenen Faktoren modelliert. Dazu wurden die Einflussfaktoren sowohl als Titel jeder Zeile als auch jeder Spalte aufgenommen und der Einfluss jeder Zeile auf Spalte durch eine Bewertung in der entsprechenden Zelle dargestellt. Dabei wurde der Einfluss von 0 bis 3 bewertet, wobei 0 keiner Beeinflussung entspricht und 3 einer sehr starken. Die Auswertung der DSM entsprechend der Passivsumme ergab, dass der Reifegrad, den ALBERS ET AL. [10] als gegenläufig zur Unsicherheit verlaufende Zielsystemdimension identifiziert haben, den am stärksten beeinflussten Faktor darstellt. Dies lässt schließen, dass der Reifegrad äquivalent zur Unsicherheit analysiert werden kann. Aus der DSM können zusätzlich die Wirkketten der Einflussfaktoren als Grafik abgeleitet werden. Ein Auszug dieser ist in Abbildung 2 dargestellt. Aus dieser Darstellung wird ersichtlich, welche Faktoren Einfluss auf die Marktunsicherheit haben, mit der ein entsprechendes Zielsystem behaftet ist.

\begin{tabular}{|l|l|l|l|l|l|l|l}
\hline Kontext der Produktentwicklung \\
\hline Dauer der Produktentwicklung [2] \\
\hline Rechtssicherheit [11]
\end{tabular}

Abbildung 2: Auszug der Wirkzusammenhänge der Einflussfaktoren für Marktunsicherheit entsprechend der DSM

Die Einflussfaktoren umfassen gemäß dem zuvor erläuterten Begriffsverständnis der Unsicherheit Wissens- und Definitionslücken hinsichtlich der Kunden und Anwender, deren Bedarfe [u.a. 12], aber auch hinsichtlich der zu betrachtenden Use-Cases [18]. Entscheidend ist 
hierbei, dass nicht nur die einzelnen Elemente unsicher sein können, sondern auch die Zusammenhänge zwischen diesen Elementen. Die Validität der Informationsbasis mit Hilfe der Annahmen über die beschriebenen Elemente getroffen werden, hat auch Einfluss auf die Unsicherheit. Ist die Aktualität der Information [23], die Stichprobengröße mit Hilfe derer die Information gesammelt und abgesichert wurde oder die Herkunft der Information nicht gewährleistet bzw. ausreichend, steigt die Unsicherheit. Weitere potentielle Unsicherheitstreiber sind Änderungen der Kundenbedürfnisse abhängig zur erwarteten Dauer der Produktentwicklung [12], die Homogenität des Markts der entsprechenden Bedürfnisse [23], die Preissensibilität [17], die Marktdynamik hinsichtlich Stabilität der Nachfrage und des Angebots [23] und der Neuentwicklungsanteil des Produkts [24].

Die Auswertung der DSM ergab weiter, dass wie bereits von ALBERS ET AL. [10] postuliert, eine ausschließliche Bewertung der Unsicherheit nur bedingt sinnvoll ist, da um Rückschlüsse hinsichtlich sich anschließender Entwicklungstätigkeiten ableiten zu können, nicht nur der Grad der Unsicherheit, sondern auch die Auswirkung dieser und die Möglichkeit der Einflussnahme bekannt sein muss. So ergab die Passivsumme, dass neben dem Reifegrad auch die Auswirkung eine hohe Passivsumme hat, also von vielen verschiedenen Faktoren stark beeinflusst wird.

\subsection{Methode zur Identifikation von Marktunsicherheiten im Zielsystem}

Nachfolgend wird ein methodischer Ansatz vorgestellt, der, auf den im vorgehenden Abschnitt vorgestellten Erkenntnissen aufbaut und Produktentwickler bei der gezielten Identifikation von mit Marktunsicherheiten behafteten Zielsystemelementen unterstützt. Um dadurch das Bewusstsein der Produktentwickler für Marktunsicherheiten und notwendigen Aktivitäten zum Umgang mit diesen zu steigern, wird das Zielsystem und seine Elemente hinsichtlich der vorliegenden Marktunsicherheit bewertet. Allerdings ist eine ausschließliche Bewertung der Marktunsicherheit nicht ausreichend, da so zwar das Bewusstsein für die Unsicherheiten gesteigert werden kann, allerdings ist es zur Unterstützung der Produktentwickler auch von Relevanz ableiten zu können, welche Aktivitäten der Produktentwicklung sich anschließen. So muss nach ALBERS ET AL. [10] außerdem die Auswirkung der Zielsystemelemente und die Möglichkeit der Einflussnahme auf das Zielsystemelement - der Härtegrad - bekannt sein. Folglich gilt es zusätzlich zu der Bewertung hinsichtlich der Unsicherheit bzw. des Reifegrades auch eine Bewertung hinsichtlich der Auswirkung der Zielsystemelemente und des Härtegrades durchzuführen. Diese drei Größen bilden damit die Dimensionen der Methode. Zielsystemelemente mit geringem Reifegrad bzw. hoher Unsicherheit stellen entsprechend der Definition Wissens- und Definitionslücken dar, mit denen es umzugehen gilt. Desto geringer der Reifegrad eines Zielsystemelementes, umso höher die Priorität. Die Auswirkung eines Zielsystemelements umfasst nach ALBERS ET AL. [10] die Konsequenzen einer Definition eines Zielsystemelements. Sie ist abhängig von den Wechselwirkungen des betrachteten Zielsystemelements im Gesamtkontext des Zielsystems. ECKERT ET AL. [25] beschreiben die Auswirkung von Änderungen als Funktion in Abhängigkeit vom Grad der Absorption bzw. vom Grad der Fortpflanzung, welche eine Änderung mit sich bringt. Absorber beschreiben dabei Elemente, welche Änderungsketten abbremsen, wogegen Multiplier diese verstärken. Die Auswirkung gibt weitere Rückschlüsse hinsichtlich der Priorität, mit der es Wissens- und Definitionslücken zu schließen gilt. Je höher die Auswirkung, umso dringlicher gilt es die Lücke zu schließen. Die kritischste Kombination stellt dabei, entsprechend der Auswirkung-ReifegradMatrix nach ALBERS ET AL. [10] ein geringer Reifegrad bei Zielsystemelementen in Kombination mit hoher Auswirkung dar. Dieser ist in Abbildung 3 als roter Bereich gekennzeichnet.

Die Bewertung des Härtegrades zeigt den Methodenanwendern die potentielle Änderbarkeit des Zielsystemelements. Zielsystemelemente, die beispielsweise aus Normen resultieren haben tendenziell einen hohen Härtegrad, da sie eingehalten werden müssen, dennoch können sie potentiell unsicher sein und eine große Auswirkung auf die Umsetzung des Produktes 
haben. Allerdings lassen sich in einem solchen Fall relativ einfach die Wissens- oder Definitionslücken schließen. Für die Validierung sind vielmehr Zielsystemelemente mit geringem Härtegrad von Relevanz, da hier eine große Einflussnahme erfolgen kann. Zusätzlich zu den beschriebenen Dimensionen entsprechend derer die Zielsystemelemente bewertet werden, ist der Kontext des Entwicklungsprojektes durch den Methodenanwender zu bewerten, da jedes Entwicklungsprojekt einzigartig und individuell ist und somit auch die Bedeutung der Unsicherheiten einen anderen Fokus hat. Von Relevanz sind hierbei beispielsweise der zeitliche Entwicklungshorizont, oder der vorliegende Neuentwicklungsanteil. Der Gesamtkontext bestimmt die Segmentierung des Diagramms und damit die Größe des roten Bereichs im Verhältnis zum grünen Bereich (vgl. Abbildung 3 links). Um das Bewusstsein der Produktentwickler für die Marktunsicherheiten zu steigern und sie bei der Priorisierung von Validierungsaktivitäten zu unterstützen werden zum Abschluss der Methodendurchführung die Ergebnisse graphisch dargestellt. Dabei werden die einzelnen bewerteten Zielsystemelemente als Punkte dargestellt. Auf der horizontalen Achse ist der Reifegrad abgebildet, auf der vertikalen die Auswirkung der Zielsystemelemente. Die Farbe der Punkte hängt vom Härtegrad des Zielsystemelements ab. Desto heller die Farbe ist, umso geringer ist der Härtegrad.
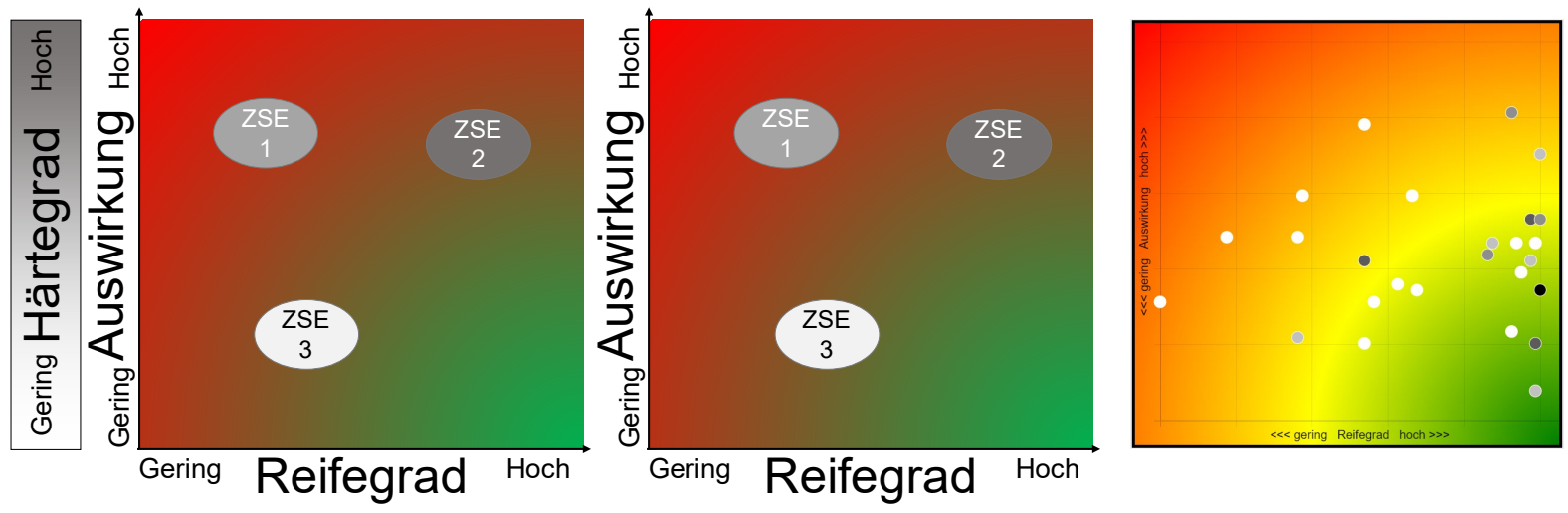

Abbildung 3: Links: Exemplarische Darstellung der Methodenergebnisse; Mitte: Auswirkung der Gesamtkontextbewertung bei höherer Unsicherheit; Rechts Visualisierung im Softwaretool

Zur Operationalisierung der Bewertung der beschriebenen Dimensionen werden bei der Methodendurchführung im Rahmen einer Diskussion die Einflussfaktoren des Reifegrads, der Auswirkung, des Härtegrads und des Gesamtkontexts durch die Methodenanwender bewertet. Die zu bewertenden Einflussfaktoren sind in Tabelle 1 dargestellt, wobei der Härtegrad direkt bewertet wird. Um eine einheitliche Bewertung durch die Produktentwickler zu ermöglichen, wurde den verschiedenen Faktoren fest Maßstäbe mit greifbaren Abstufungen hinterlegt.

Tabelle 1: Zu bewertende Einflussfaktoren auf Reifegrad, Auswirkung und Gesamtkontext der Produktentwicklung

\begin{tabular}{|c|c|c|c|}
\hline Reifegrad & Auswirkung & Härtegrad & Gesamtkontext \\
\hline $\begin{array}{l}\text { Bekanntheit des adressierten } \\
\text { Kunden-/Anwendernutzen }\end{array}$ & $\begin{array}{l}\text { Auswirkung des Zielsystemele- } \\
\text { ments auf Kundennutzen }\end{array}$ & Härtegrad & Entwicklungshorizont \\
\hline $\begin{array}{l}\text { Bekanntheit der relevanten Kun- } \\
\text { den und Anwender }\end{array}$ & $\begin{array}{l}\text { Auswirkung des Zielsystemele- } \\
\text { ments auf Anwendernutzen }\end{array}$ & & Markthomogenität \\
\hline $\begin{array}{l}\text { Bekanntheit des relevanten } \\
\text { Use-Cases }\end{array}$ & $\begin{array}{l}\text { Auswirkung des Zielsystemele- } \\
\text { ments auf Produktentwicklung }\end{array}$ & & Preissensibilität \\
\hline $\begin{array}{l}\text { Bekanntheit des Zusammenhangs } \\
\text { zwischen Zielsystemelement und } \\
\text { Kunden-/Anwendernutzen }\end{array}$ & $\begin{array}{l}\text { Auswirkung bei Änderung des } \\
\text { Zielsystemelements }\end{array}$ & & Marktdynamik \\
\hline Validität der Informationen & & & Neuentwicklungsanteil \\
\hline
\end{tabular}


Um die Bewertungen der einzelnen Faktoren in ein Gesamtmodell zu übertragen, bedarf es einer geeigneten Metrik. Diese soll möglichst viele Informationen der Einzelbewertung beinhalten und valide Informationen hinsichtlich der Gesamtbewertung der verschiedenen Dimensionen bieten. Bei einer Multiplikation der Faktoren sind die Ergebnisse nicht gleichmäßig über den Definitionsbereich verteilt und es führen deutlich mehr Kombinationen zu niedrigen Ergebnissen als zu hohen. Deswegen wird im Rahmen dieser Methode auf den Least Square Ansatz aus der Regression zur Berechnung der Darstellungsdimensionen zurückgegriffen. Dabei wird jedem Einflussfaktor eine ideale Ausprägungsstufe zugeordnet. Diese entspricht dem unkritischsten Fall. Um die Darstellungsdimension zu erhalten wird nun die Differenz zwischen der Bewertung des Faktors und der idealen Ausprägung gebildet und anschließend quadriert, um das Abstandquadrat zu erhalten. Die Summe der Abstandsquadrate ergeben die Darstellungsdimension für Reifegrad, Auswirkung, Härtegrad und Gesamtkontext.

$$
\text { Darstellungsdimension }=\sum\left(\text { Faktor }_{i, \text { Bewertet }}-\text { Faktor }_{i, \text { Ideal }}\right)^{2}
$$

Formel 1: Berechnung der Darstellungsdimension aus den bewerteten Einflussfaktoren

Die Bewertung des Zielsystems stellt keinen einstufig ablaufenden Prozess dar, sondern vielmehr ist die initiale Bewertung kontinuierlich im Verlauf der Produktentwicklung zu überprüfen. Abhängig vom aktuellen Wissens- und Definitionsstand wird die Bewertung als Teil des iterativen Prozesses der Produktentwicklung angepasst. Neu entstehendes Wissen verändert die Bewertungen hinsichtlich der vorliegenden Unsicherheiten und Auswirkungen und es können neue Aktivitäten abgleitet werden.

\subsection{Initiale Evaluierung der Methode zur Identifikation von Marktunsicherheiten}

Die in Abschnitt 4.2 vorgestellte Methode zur Identifikation von Marktunsicherheiten wurde im Zuge eines Entwicklungsprojekts der Hekatron Vertriebs $\mathrm{GmbH}$ angewendet und evaluiert. Dazu wurde eine Anwendungs- und Erfolgsevaluierung durchgeführt, wobei zur Vorbereitung alle Zielsystemelemente einheitlich formuliert und in das entwickelte Softwaretool implementiert wurden. Im Zuge der Anwendungsevaluierung wurde die Verständlichkeit der Methode und des Tools interviewbasiert analysiert. Weiter wurde der wahrgenommene Nutzen der Methodenanwender im Vergleich zum Aufwand bewertet, wobei der Nutzen der Methode im Verhältnis zum Aufwand der Methodendurchführung als hoch bewertet wurde und auch die Verständlichkeit der Methode und des Tools sehr gut bewertet wurde. Insbesondere die Einflussfaktoren und die Bewertungsstufen wurden positiv bewertet. Auch die Erfolgsevaluierung wurde interviewbasiert durchgeführt. Fokus hierbei war einerseits die Eignung kritische Zielsystemelemente zu identifizieren und entsprechende Aktivitäten abzuleiten und andererseits das Bewusstsein für Marktunsicherheiten. Vor der Durchführung der Methodendurchführung wurden von einem Fachexperte 12 von 30 Zielsystemelementen als unsicher bewertet, während der Methodenanwender lediglich 7 Elemente entsprechend kennzeichnet. Durch die gemeinsame Bewertung mit dem Tool wurden 15 Zielsystemelemente identifiziert, die mit Marktunsicherheiten behaftet sind. Dabei gab es eine 100 prozentige Überschneidung mit denen, die vor dem Workshop durch den Experten identifiziert wurde. Durch die Methode konnten noch drei weitere unsichere Zielsystemelemente identifiziert werden. Beim Methodenanwender wurden, im Verhältnis zur Bewertung vor der Methodendurchführung, 10 weitere unsichere Elemente identifiziert, 2 kritische Elemente nach der Methodendurchführung jedoch als sicher bewertet werden. Die subjektiv wahrgenommene Unsicherheit des Methodenanwenders war vor und nach Methodenanwendung gleich, gestiegen ist jedoch die Konfidenz, die kritischen Zielsystemelemente identifiziert zu haben. Ebenso konnte der wahrgenommene Handlungsbedarf und die Konfidenz, richtige Validierungsaktivitäten auszuwählen gesteigert werden. 


\section{Diskussion}

Die in dieser Veröffentlichung vorgestellten Ergebnisse haben einen konzeptionellen Charakter. Zwar wurde bereits eine erste Evaluation der Methode durchgeführt, jedoch gilt es nachfolgend die gesammelten Ergebnisse durch weitere Validierungsstudien zu vertiefen. Im Rahmen dieser sich anschließenden Validierungsstudien soll erörtert werden, ob die richtigen Einflussfaktoren ausgewählt wurden und die richtigen Wirkzusammenhänge identifiziert wurden. Zwar wurde diese Auswahl auf Basis einer Literaturrecherche und einer Design Structure Matrix durchgeführt, jedoch muss angeführt werden, dass die DSM einen eher subjektiven Charakter hat. Aktuell ist keine Gewichtung der Einflussfaktoren hinterlegt, sondern alle Faktoren bzw. die Abstandquadrate werden lediglich aufsummiert. Eventuell ist dieser lineare Zusammenhang jedoch nicht zutreffend. Statt, dass, wie beispielsweise bei einer FMEA, die Einzelbewertungen zur Ermittlung der Gesamtbewertung multipliziert werden, also das Produkt der Bewertungen der einzelnen Einflussfaktoren gebildet wird, wurde im Zuge der Erstellung der Metrik sich dafür entscheiden die Fehlerquadrate der einzelnen Faktoren aufzusummieren, damit die einzelnen Faktoren ein größeres Gewicht erhalten. Hier gilt es die Methode in weiteren Projekten anzuwenden und die Ergebnisse von Experten bewerten zu lassen, um festzustellen, ob die richtigen Unsicherheiten identifiziert werden. Weiter kann es passieren, dass je nach Projektreifegrad oder Produktart manche Einflussfaktoren nicht bewertbar sind, da die Ausprägungsstufen nicht zutreffend sind. Auch hierzu sollen weitere Untersuchungen durchgeführt und die Bewertungsstufen eventuell angepasst werden. Übergreifend lässt sich jedoch feststellen, dass die Methode in einer ersten Evaluierung bereits positive Ergebnisse gezeigt hat und seitens des Methodenanwenders ein erhöhter Handlungsbedarf zum Umgang mit den Marktunsicherheiten nach der Methodendurchführung festgestellt wurde.

\section{Zusammenfassung und Ausblick}

Im Zuge dieser Veröffentlichung wurden zunächst, aufbauend auf einer Literaturrecherche, Einflussfaktoren zur Charakterisierung von Markunsicherheit identifiziert. Diese wurden nachfolgend durch eine Design Structure Matrix analysiert und somit die Wirkzusammenhänge zwischen den Einflussfaktoren charakterisiert. Darauf aufbauend wurde im Anschluss eine Methode erarbeitet, die das Bewusstsein von Produktentwicklern hinsichtlich vorliegender Marktunsicherheit steigert und gleichzeitig dabei unterstützt gezielt Validierungsaktivitäten abzuleiten. Diese Methode baut auf den Zielsystemdimensionen Reifegrad, Auswirkung und Härtegrad auf. Im Zuge der Methodendurchführung werden durch die Produktentwickler sämtliche Zielsystemelemente entsprechend der Einflussfaktoren bewertet, und damit Werte für die Dimensionen errechnet. Das Ergebnis wird abschließend graphisch dargestellt. Im Zuge einer ersten Evaluation konnte der Nutzen nachgewiesen werden. So eignet sich die Methode dazu mit einen angemessenen Aufwand Marktunsicherheiten innerhalb des Zielsystems zu identifizieren. Nachfolgend gilt es die Evaluation zu vertiefen und in weiteren Projekten mit mehr Teilnehmern durchzuführen, um das Feedback zu erweitern. Außerdem soll eine Verknüpfung zu Validierungsmethoden hergestellt werden, damit auf Basis der durchgeführten Bewertung die Methodenanwender gezielt Vorschläge für Methoden unterbreitet bekommen, mit denen sie die Unsicherheiten reduzieren können.

\section{Literaturverzeichnis}

[1] The Standish Group: Chaos Report. Massachusetts, 2015.

[2] Browning, Tyson R.: Modeling and analyzing cost, schedule, and performance in complex system product development. Massachusetts Institute of Technology, Sloan School of Management. Dissertation 1998.

[3] Song, Young-Woo; Herzog, Michael; Beate Bender: Understanding the Initial Requirements Definition in Early Design Phases. In: Proceedings of the Design Society: International Conference on Engineering Design Vol. 1 (2019), Nr. 1, S. 3751-3760. 
[4] Cao, Yong; Chen, Ren-song; Zhao, Li; Nagahira, Akio: Impact analysis of FFE practices of New Product Development in Japanese companies. In: 2008 International Conference on Management Science and Engineering 15th Annual Conference Proceedings (2008), S. 1449-1456.

[5] Gausemeier, Jürgen: Zukunftsorientierte Unternehmensgestaltung. In: ZWF Zeitschrift für wirtschaftlichen Fabrikbetrieb Vol. 104, (2009), Nr. 7-8, S. 623-626.

[6] Albers, Albert; Behrendt, Matthias; Klingler, Simon; Reiß, Nicolas; Bursac, Nikola: Agile product engineering through continuous validation in PGE - Product Generation Engineering. In: Design Science Vol. 3, (2017).

[7] Heimicke, Jonas; Niever, Manuel; Zimmermann, Valentin; Klippert, Monika; Marthaler,Florian; Albers, Albert: Comparison of Existing Agile Approaches in the Context of Mechatronic System Development: Potentials and Limits in Implementation. In: Proceedings of the Design Society: International Conference on Engineering Design Vol. 1 (2019), Nr. 1, S. 2199-2208.

[8] Sutcliffe, Alistair: A conceptual framework for requirements engineering. In: Requirements engineering Vol. 1 (1996), Nr. 3, S. 170-189.

[9] Albers, Albert: Five Hypotheses about Engineering Processes and their Consequences. In: Proceedings of the TMCE Vol. 12 (2010), S. 343-356.

[10] Albers, Albert; Lohnmeyer, Quentin; Ebel, Björn: Dimensions of objectives in Interdisciplinary Product Developement Projects. In: Proceedings of the 18th International Conference on Engineering Design (ICED 11) Vol. 2, (2011), DS 68-2, S. 256-265.

[11] de Weck, Olivier; Eckert, Claudia; Clarkson, P. John: A classification of uncertainty for early product and system design. In: Proceedings of the International Conference on Engineering Design (ICED) Vol. 42, (2007).

[12] Ebert Christof; De Man, Jozef: Requirements Uncertainty: influencing factors and concrete improvements. In: Proceedings of the 27th international conference on Software engineering, (2005), S. 553-560.

[13] Mcmanus, Hugh; Hastings, Daniel Edga: A framework for understanding uncertainty and its mitigation and exploitation in complex systems. In: Engineering Systems Symposium, (2004).

[14] Wynn, David C.; Grebici, Khadidja; Clarkson, P. John: Modelling the evolution of uncertainty levels during design. In: International Journal on Interactive Design and Manufacturing Vol. 5, (2011), Nr. 3, S. 187.

[15] Koppelmann, Udo: Produktmarketing: Entscheidungsgrundlagen für Produktmanager: Berlin Heidelberg: Springer-Verlag, 2013.

[16] Logue, Kevin; McDaid, Kevin: Handling Uncertainty in Agile Requirement Prioritization and Scheduling Using Statistical Simulation. In: Agile Development Conference, (2008), S. 73-82.

[17] Verworn, Birgit; Herstatt, Cornelius: Strukturierung und Gestaltung der frühen Phasen des Innovationsprozesses. In: Herstatt, Cornelius; Verworn, Birgit(Hrsg.): Management der frühen Innovationsphasen: Grundlagen - Methoden - neue Ansätze. 2. Aufl. Wiesbaden: Betriebswirtschaftlicher Verlag Dr. Th. Gabler, 2007, S. 112-134.

[18] Albers, Albert; Klingler, Simon; Wagner, Daniel: Prioritization of Validation Activities in Product Developement Process. In: 13th International Design Conference Vol. 13 (2014), S. 81-90.

[19] Hering, Ekbert; Schloske, Alexander: Fehlermöglichkeits- und Einflussanalyse: Methode zur vorbeugenden, systematischen Qualitätsplanung unter Risikogesichtspunkten. Wiesbaden: Springer Vieweg, 2019.

[20] Engelhardt, Roland; Birkhofer, Herbert; Kloberdanz, Hermann; Mathias, Johannes: Uncertainty-Mode-and Effects-Analysis-an Approach to Analyze and Estimate Uncertainty in the Product Life Cycle. In: Proceedings of ICED 09, the 17th International Conference on Engineering Design Vol. 2. (2009), S. 191-202.

[21] Wiebel, Marion; Eifler, Tobias; Mathias, Johannes; Kloberdanz, Hermann; Bohn, Andrea; Birkhofer, Herbert: Modellierung von Unsicherheit in der Produktentwicklung. In: Jeschke, Sabina; Jakobs, Eva-Maria; Dröge, Alicia: Exploring Uncertainty: Ungewissheit und Unsicherheit im interdisziplinären Diskurs. Wiesbaden: Springer Fachmedien Wiesbaden, 2013, S. 245-269.

[22] Blessing, Lucienne T. M.; Chakrabarti, Amaresh: DRM: A design research methodology. London: Springer London, 2009.

[23] Gericke, Kilian; Meißner, Moritz; Paetzold, Kristin: Understanding the context of Product Developement. In: Proceedings of the 19th International Conference on Engineering Design (ICED13) Design for Harmonies, Vol. 3: Design Organisation and Management, (2013), S. 191-200.

[24] Albers, Albert; Bursac, Nikola; Wintergerst, Eike: Produktgenerationsentwicklung - Bedeutung und Herausforderungen aus einer entwicklungsmethodischen Perspektive. In: Stuttgarter Symposium für Produktentwicklung, (2015).

[25] Eckert, Claudia; Clarkson, P. John; Zanker, Winfried: Change and customisation in complex engineering domains. In: Research in Engineering Design Vol. 15 (2004), Nr. 1, S. 1-21. 